Journal für Mobilität und Verkehr

ISSN 2628-4154

www.dvwg.de

\title{
Bieterwettbewerb Klimaneutralität - Grenzüberschreitende Verkehre in realitätsfernen politischen Programmen
}

\author{
Prof. Dr. Dr. Franz Josef Radermacher \\ Forschungsinstitut für anwendungsorientierte Wissensverarbeitung/n (FAW/n), Lise-Meitner-Str. 9, 89081 Ulm, Deutschland
}

\begin{abstract}
Der Text betrachtet Verkehr und Mobilität als Teil der internationalen Energie- und Klimathematik. Er beschreibt, weshalb der in Deutschland verfolgte Zugang zu den Themen in seiner auf das Nationale fixierten Betrachtungsweise zur Lösung der bestehenden Probleme fast nichts beitragen kann. Leider werden durch diese Politikansätze aber erhebliche Kollateralschäden verursacht, die die Mobilitätszukunft in Deutschland und Europa erheblich belasten und zu großen Wohlstandsverlusten führen können. Wie problemadäquate, alternative Ansätze aussehen könnten, wird anhand der Logik von Global Energy Solutions e. V. beschrieben.
\end{abstract}

Schlagwörter/Keywords: CO2 Recyclierung, Globale Lösungsansätze, Grüner Strom, Grüner Wasserstoff, Klimanationalismus, Klimaneutralität, Negativemissionen, Synthetische Kraftstoffe, Wasserstoff-Hochlauf

\section{Einleitung}

Die Zukunft der Mobilität und des Verkehrs in Deutschland / in der DACH-Region ist ein Thema, das mit hoher Emotionalität, einem teils starken nationalen Fokus und einem teils sehr verengten Blick auf technische Optionen angegangen wird. Zur Lösung der Weltklimaproblematik trägt ein solcher Zugang wenig bei. Die Schweiz operiert dabei in vielen Dimensionen viel weniger dogmatisch als Deutschland. Der vorliegende Text analysiert einige Zusammenhänge.

\section{Klima-Nationalismus}

Deutschland fokussiert sich im Kampf gegen den Klimawandel zu sehr auf nationale Ziele (Klima-Nationalismus). Diese sind im globalen Kontext jedoch wenig relevant. Dieser Fokus führt zu ungünstigen Strategien, etwa in den Bereichen grüner Strom, grüner Wasserstoff und synthetische Kraftstoffe. Alle Überlegungen sind beherrscht durch Knappheit und zu hohen Kosten. Weil man in Deutschland selber produzieren will, was klugerweise importiert werden sollte. So wie bisher Energie zu 70 \% importiert wird.

Das Thema Klimaschutz ist extrem komplex. Die weltwei- ten $\mathrm{CO}_{2}$-Emissionen wachsen weiter. Der Paris-Vertrag beinhaltet zwar ambitionierte Zielsetzungen, aber keine dazu passenden Verpflichtungen und Maßnahmen. Die negative Dynamik im Klimabereich resultiert aus den nachvollziehbaren wirtschaftlichen Bestrebungen vieler ärmerer Länder in Richtung eines nachzuholenden Wohlstands.

China gibt die Richtung vor, stößt aber in der Folge ein Drittel der weltweiten $\mathrm{CO}_{2}$ Emissionen aus und erhöht diese weiter. Die Weltbevölkerung wächst parallel dazu mit hohem Tempo. Bis 2050 werden etwa 2,5 Milliarden Menschen hinzukommen, jedes Jahr einmal die Bevölkerungsanzahl der Bundesrepublik.

Die deutsche und europäische Klimapolitik beschäftigt sich mit diesen Themen nur wenig. Wir sind primär mit der Absenkung unserer eigenen $\mathrm{CO}_{2}$-Emissionen beschäftigt. Für das Weltklima ist das nur wenig relevant, kostet aber all unsere Aufmerksamkeit und enorme finanzielle und intellektuelle Ressourcen. Wir setzen voll auf Elektromobilität, nicht auf klimaneutrale synthetische Kraftstoffe für Pkw, u. a. für die weltweite Bestandsflotte. Atomkraft wird pauschal abgelehnt ebenso wie die Abscheidung und Nutzung von $\mathrm{CO}_{2}$ aus Industrieanlagen und Kohlekraftwerken. 


\section{reFuels und Kostenfragen}

Auf der Welt fahren ungefähr 1,3 Milliarden Fahrzeuge mit Verbrennungsmotoren, der größte Teil davon im Bereich individueller Mobilität, etwa 300 Millionen Fahrzeuge sind (schwere) LKWs. Es ist damit zu rechnen, dass über die nächsten Jahrzehnte noch viele weitere derartige Fahrzeuge hinzukommen werden und im Durchschnitt mindestens 1,5 Milliarden Verbrennerfahrzeuge fahren werden, dies nicht zuletzt wegen des weiter hohen Bevölkerungswachstums und der verbreiteten Armut und der legitimen Erwartungen an Entwicklung. In Deutschland sind es heute etwa 47 Millionen solcher Fahrzeuge, davon etwa 65\% Benziner und etwa 35\% Diesel-Fahrzeuge. Der gesamte weltweite Emissionsumfang in diesem Bereich liegt bei etwa 5 Milliarden Tonnen $\mathrm{CO}_{2}$ pro Jahr. Die Emissionen bei Flugzeugen und Schiffen liegen in Summe bei vielleicht einem Drittel der Emissionen im Bereich der Fahrzeuge mit Verbrennungsmotoren. Es wäre zur Erreichung des $2^{\circ} \mathrm{C}$-Ziels sehr hilfreich, wenn man mit synthetischen Kraftstoffen, reFuels, zu einer Alternative zum Status Quo käme. Vernünftigerweise sollten aus diesen Kraftstoffen auch Steuereinnahmen generiert werden, wie es heute auch der Fall ist, denn das Straßensystem muss finanziert werden. Trotzdem sollten die Kosten unter 2 Euro pro Liter bleiben können, dann wäre ein Wechsel in Richtung synthetischer Kraftstoffe für letztlich alle Verbrenner auf der Erde eine realistische Option.

Untersuchungen aus dem Bereich der Mineralölwirtschaft und vieler thematisch tangierter Verbände, wie insbesondere die "Frontier-Studie" und Untersuchungen des Verbandes der Automobilindustrie (VDA), wie Untersuchungen im Umfeld des Autors, die vor allem von führenden Persönlichkeiten von Global Energy Solutions e.V. begleitet wurden und auf eine breite Literatur zum Thema wie auf eigenen industriellen Erfahrungen aufbauen, zeichnen bezüglich der Kostensituation folgendes Bild:

In den großen Sonnenwüsten der Welt lassen sich Preise für grünen Strom von unter 2 Cent pro kWh am Übergabepunkt zur Elektrolyse erreichen. Tatsächlich liegen die Preise heute teilweise sogar schon deutlich darunter. Damit kommt man auf einen Preis für grünen Wasserstoff von etwa 1 Euro pro Tonne. Die Weiterverarbeitung des grünen Wasserstoffs zu (grünem) Methanol und der Transport nach Deutschland führen zu einem Preis pro Doppelliter (entspricht energetisch etwa 1 Liter Benzin) von etwa 1 Euro. Bei Weiterverarbeitung zu Methanolbenzin und Zuschlag aller heute gezahlten Steuern bleibt man unter 2 Euro pro Liter. Würde dieser Kraftstoff regulativ als klimaneutral akzeptiert, käme man in eine tragfähige Größenordnung im Verhältnis zum Status Quo, da $\mathrm{CO}_{2}$-Abgaben auf diese Kraftstoffe entfallen sollten/würden. Vor allem müssen weder die Fahrzeuge umgebaut oder ersetzt noch die Infrastruktur ersetzt wer- den.

Diese Situation sieht eigentlich sehr vielversprechend aus. Die Zusammenarbeit z. B. von Afrika und Europa würde so die europäischen und die afrikanischen Klimaziele vergleichsweise preiswert zu erreichen erlauben, was keiner der Akteure alleine schaffen könnte. Für Europa ergeben sich in diesem Kontext attraktive industriepolitische Perspektiven in einem schwierigen Umfeld, das immer stärker durch Rivalitäten zwischen den USA und China bestimmt wird. Allerdings ist der vor den Beteiligten liegende Weg lang. Aktuell wird er bzgl. der Situation in Marokko durch den Westsahara-Konflikt überlagert.

Insgesamt erweist es sich als schwierig, den beschriebenen Weg umzusetzen. Das erste Projekt der beschriebenen Art realisieren Siemens Energy und Porsche mit Förderung des Bundeswirtschaftsministeriums in Chile (Projekt Haru Oni), weil die infrastrukturellen Voraussetzungen in Chile besser sind als in Nordafrika. Die Firma Obrist aus Vorarlberg verfolgt einen Ansatz mit einem Fahrzeug, das einen Methanolmotor mit einer kleinen Batterie koppelt. Das Methanol wird klimapositiv in der namibischen Wüste produziert. Das Projekt wird mit Förderung des Bundesforschungsministeriums in Deutschland realisiert. Das ist die eine Seite.

Auf der anderen Seite wollen diverse private und gesellschaftliche Akteure lieber grüne Projekte vor Ort anschieben, als auf weltweite Kooperationen zu setzen. Diese sollen das Geld im Land halten und neue Arbeitsplätze schaffen. Wo nötig, soll Geld zugeschossen werden, entweder direkt vom Staat oder von den Bürgern, denen dies gegebenenfalls regulativ aufgezwungen werden soll. Gegen Kooperationen mit Partnern in Afrika werden Argumente vorgebracht wie neuer Kolonialismus, Blockierung einer erhofften Energiewende in diesen Ländern, Risiken für die Versorgungssicherheit bei uns etc. Aus Sicht des Autors ist das "Thema verfehlt". Wie so oft scheint es nicht zu interessieren, wie wir bezüglich der weltweiten Arbeitsteilung mit unseren Partnern, z. B. in Nordafrika, umgehen (siehe EEG als nicht-tarifäres Handelshindernis). Diese Haltung kann international noch viele Konflikte nach sich ziehen. Die vielfältigen Potentiale weltweiter Kooperation werden demgegenüber offenbar völlig unterschätzt.

Die Preise für Wasserstoff bei Produktion in Europa liegen bei fossil-basiertem Wasserstoff bei etwa 1-1,50 Euro pro $\mathrm{kg}$. Direktreduktion mit solchem Wasserstoff ist im Bereich Stahl eine konkurrenzfähige Alternative zum heutigen Einsatz von Koks/Kohle in diesem Bereich. Bei der sogenannten grünen Variante (Direktreduktion mit grünem Wasserstoff) liegt man in Deutschland bei 4-6 Euro und mehr pro Kilogramm Wasserstoff. Will man die daraus resultierenden Differenzkosten von günstig gerechnet 100 Euro pro Tonne Stahl (bei 40 Millionen Tonnen Produktion in Deutschland 
pro Jahr) gegenüber den heutigen Kosten von etwa 400 Euro pro Tonne Stahl abdecken, liegt man jährlich bei 4 Milliarden Euro. Die deutsche Wasserstoffstrategie setzt nach heutigen Planungen bis 2030 Fördermittel von 7 Milliarden Euro insgesamt für den Wasserstoff-Hochlauf ein. Wegen Engpässen bei grünem Strom ist der entsprechende grüne Wasserstoff in dieser Menge hier absehbar gar nicht produzierbar, schon gar nicht bezahlbar. Weder mengenmäßig noch von der Finanzierung her erscheint dieser Weg als zukunftssicher.

Den Zuständigen in Deutschland sind diese Probleme in unterschiedlichem Umfang bekannt. Entscheidend sind der Engpass bei grünem Strom und seine hohen Kosten. Der letzte Punkt erfordert einerseits den Außenschutz über das EEG als nicht-tarifäres Handelshindernis, andererseits Vorgaben gegen „ineffiziente“ Nutzungen des wenigen verfügbaren grünen Stroms, der mit Blick auf das Erreichen der Klimaziele an vielen Stellen gebraucht wird. Dieser soll nicht durch Nutzungskonkurrenz noch teurer werden.

Wenn man also auf die erneuerbare Energie vor Ort setzt, wird man alles tun müssen, um Ineffizienzen in der Nutzung des grünen Stroms zu verhindern. Das erklärt die massive Ablehnung vieler politischer Akteure in diesem Umfeld gegenüber reFuels / synthetischen Kraftstoffen. Deren Einsatz soll insbesondere wegen der hohen Umwandlungsverluste im Verhältnis zum Direkteinsatz von (grünem) elektrischem Strom beim Elektroauto verhindert werden. Deshalb tun sich alle diejenigen, die für die Nutzung von reFuels im Individualverkehr werben, zurzeit in Deutschland schwer. Sie werden mit dem „absurden“ Argument als den grünen Wasserstoff als den „Champagner der Energiewende“ konfrontiert, wobei die zum "Champagner" führende Knappheit aber selber regulativ erzeugt wird. Die Bewertung würde ganz anders ausfallen, wenn die Produktion der reFuels an geeigneten Standorten im Ausland unter Berücksichtigung von Nachhaltigkeitsaspekten erfolgt. Oder wenn statt grünem Wasserstoff sogenannter blauer Wasserstoff (Herstellung mit Gas, Abfangen und Verpressen des $\mathrm{CO}_{2} / \mathrm{CCS}$ ) als „grün“ akzeptiert werden würde, was der Autor empfiehlt.

Zu wenig beachtet wird bisher in vielen Diskussionen, dass die weltweite Zusammenarbeit extrem wichtig ist und dass über die weltweite Zusammenarbeit der grüne Strom so preiswert produziert werden kann, dass Effizienznachteile keine Bedeutung mehr haben und die Endprodukte, z. B. synthetische Kraftstoffe, in die Preisdimensionen der heutigen fossilen Kraftstoffe fallen. Weltweit muss man da hin, wenn man das Klimaproblem lösen will. Bei einer richtigen Ausgestaltung der Strategie lösen sich also diese Effizienzschwierigkeiten auf.

Die entscheidende These ist hier: Wir können die weltweiten Energie- und Klimaprobleme nur lösen, wenn wir ein drittes Standbein haben, nämlich neben dem grünen Strom und dem grünen Wasserstoff auch synthetische grüne Kraftstoffe zu tragfähigen Preisen. Indem wir diese international produzieren, nutzen wir eine vernünftige internationale Arbeitsteilung. Wir werden als Deutschland mit Technologieexporten, z. B. Elektrolyseure, viel Geld verdienen, ebenso mit CCU/CCS-Technologie. Das ist für uns und Europa ein guter Ansatz. Wir sollten die Produktion von grünem Strom, Wasserstoff und synthetischen Kraftstoffen dort geschehen lassen - ja sogar wollen und aktiv fördern -, wo es sich lohnt, preiswert möglich ist und wo es unkompliziert ist. Deutschland hätte einen Vorteil davon, wenn man einen solchen Weg wählt, die Welt ebenfalls. Das gilt im Besonderen für viele Entwicklungs- und Schwellenländer und beinhaltet auch erhebliche positive Potentiale im Hinblick auf die Bewältigung der Bevölkerungsfrage.

\section{$\mathrm{CO}_{2}$-Recyclierung}

Der beschriebene Weg zu reFuels beinhaltet eine weitere wichtige Dimension. Grünes Methanol und Methan entstehen aus grünem Wasserstoff über Synthese mit CO2 bzw. CO. CO gewinnt man aus $\mathrm{CO} 2$. Um dieses $\mathrm{CO} 2$ zu erhalten, bieten sich verschiedene Wege an. In der deutschen Debatte will man wegen der offensichtlichen Konzentration auf Stromlösungen vor Ort vor allem $\mathrm{CO}_{2}$ aus solchen industriellen Prozessen nutzen, die nicht gut elektrifizierbar sind, also z. B. Kalk und Zement. Wegen der zu erwartenden massiven Bautätigkeit in vielen Entwicklungs- und Schwellenländern, insbesondere Afrika, gibt es hier ein großes Potential. Positiv gesehen wird biogenes Material, dass aber aus Sicht des Autors wegen der zunehmenden Weltbevölkerungsprobleme zukünftig immer weniger für diesen Zweck verfügbar sein wird. Eine weitere (bis heute sehr teure) Option ist Direct Air Capture. Sollte sich der Strompreis in Sonnenwüsten am Übergabepunkt zur Nutzung in Richtung 1 Cent/kWh bewegen und sollten weitere erhebliche Kostensenkungen bei den eigentlichen Anlagen gelingen, kann dies interessant werden. Vielleicht gelingen Preise unter 50 Euro pro Tonne $\mathrm{CO}_{2}$, aber das wird, wenn überhaupt, noch sehr lange dauern. Auch bleibt die Frage, was mit dem $\mathrm{CO}_{2}$ geschehen soll, wenn man den Umfang von Direct Air Capture immer weiter ausdehnt. Ein Erfolg würde es natürlich sehr vereinfachen, die Weltklimafrage anzugehen und in all diesen Fragen langfristig die $\mathrm{CO}_{2}$-Konzentration in der Atmosphäre sogar wieder zu senken. Hier würde sich zugleich eine naheliegende Obergrenze für Preise auf $\mathrm{CO}_{2}$-Emissionen ergeben, die uns die heute teilweise diskutierten, wohlstandbedrohenden Preise von vielen 100 Euro ersparen würde.

Ganz wichtig ist dem Autor ein weiterer Aspekt, der in der deutschen Diskussion teilweise vehement bekämpft wird. Nämlich die offenbar vernünftige und oft relativ preiswerte Lösung, $\mathrm{CO}_{2}$ in großem Stil in Industrieprozessen, z. B. bei 
der Stahlproduktion und auch bei fossilen Kraftwerken abzufangen und z. B. zur Herstellung von synthetischen Kraftstoffen zu nutzen. Dies z. B. mit dem naheliegenden Argument, dass jede vermiedene Tonne $\mathrm{CO}_{2} \mathrm{Sinn}$ macht. Und wenn man $\mathrm{CO}_{2}$ in der Stahlproduktion einspart, dass nachher bei der Nutzung von Methanolbenzin in Autos in die Atmosphäre geht, hat man den Emissionsumfang zumindest halbiert. Warum? Statt der $\mathrm{CO}_{2}$-Emissionen bei der Stahlproduktion und der $\mathrm{CO}_{2}$-Emissionen durch die Nutzung fossiler Treibstoffe im Auto hat man (volumenmäßig) nur noch die Emissionen der Verbrennung der reFuels, die den Emissionen im Auto aus fossilen Kraftstoffen entsprechen. Diese $\mathrm{CO}_{2}$-Emissionen sind jetzt aber das $\mathrm{CO}_{2}$, das vorher schon durch die Stahlproduktion in die Atmosphäre gelangt wäre, jetzt aber abgefangen wurde, um die reFuels damit herzustellen. Ist das reFuels vom Typ klimaneutrales Gas, kann man es direkt für die klimaneutrale Stahlproduktion nutzen. Dann bleibt das $\mathrm{CO}_{2}$ in technischen Kreisläufen und gelangt nicht mehr in die Atmosphäre. Das ist dann Mehrfach-Recyclierung. Bei mehrfacher Recyclierung, die ein zentrales Element des hier vertretenen Lösungsweges ist, kann man vielleicht eine Reduktion auf $20 \%$ der bisherigen $\mathrm{CO}_{2}$-Emissionen erreichen.

Es lässt sich in der angedeuteten Weise ein geschlossener Kreislauf organisieren. Wir würden dann auf drei Beinen stehen und hätten insgesamt eine deutlich verbesserte Ausgangssituation zur Lösung der globalen Energie- und Klimaprobleme herbeigeführt. Es ist wichtig, dass dies in einer Weise gelingt, dass die auf fossilen Energien basierenden Industrien nicht ökonomisch kollabieren. Das würde nämlich zu massiven ökonomischen Verwerfungen führen, $u$. $U$. auch zu Auseinandersetzungen zwischen den Staaten bzw. zu Bürgerkrieg innerhalb von Staaten, denen die bisherigen Haupteinnahmen wegfallen. Das Gegenteil sollte passieren. Es soll für die entsprechenden leistungsstarken Akteure attraktiv sein, die Transformation zu einer neuen Energiesparte zu ihrem eigenen Vorteil zu gestalten, vor allem auch zu finanzieren und ihre eigenen Geschäftsmodelle anzupassen. Warum wird ein solcher Ansatz teilweise trotzdem bekämpft? Verwendet werden Argumente wie ein befürchteter Lock-in Effekt für die Kohle. Während man einen Kreuzzug gegen die Kohle führt, sollen Kohlekraftwerke nicht in Richtung einer (teilweisen) Klimaneutralität geführt werden. Egal, ob bei Verzicht auf diesen Weg vielleicht alle Chancen untergraben werden, Energiewohlstand für viele Menschen in Entwicklungs- und Schwellenländern mit Anliegen des Klimaschutzes zu verknüpfen.

\section{Grüner Strom}

Über die letzten Jahrzehnte haben sich die Möglichkeiten stark verbessert, grünen Strom, also erneuerbare Energie in Stromform, zu produzieren, vor allem über große Instal- lationen von Photovoltaikanlagen und über große Onshoreund Offshore-Windkraft. Besonders interessant sind - auch unter Entwicklungsaspekten - die Potentiale in den großen Sonnenwüsten der Welt.

Die Welt braucht preiswerten grünen Strom in riesigen Mengen, wenn eine Klimakatastrophe noch verhindert werden soll. Der Autor rechnet für 2050 mit bis zu 400.000 TWh für die ganze Welt. Dabei wird das weitere Wachstum der Weltbevölkerung, der erhoffte weitere Wohlstandszuwachs (gerade auch in ärmeren Ländern) und die geringere Energieeffizienz in ärmeren Ländern eingerechnet. Berücksichtigt sind auch die Energieverluste, wenn grüner Strom in andere Nutzungsformen (z. B. e-Fuels) überführt wird. Die gesamte Stromproduktion in Deutschland, inklusive Strom aus Kohle, liegt heute bei etwa 700 TWh. Die deutsche Bundesforschungsministerin Anja Karliczek will bis 2040 etwa 800 TWh des deutschen Energiebedarfs aus grünem Wasserstoff decken. Sie hat jüngst den Potenzialatlas vorgestellt, der alleine in Westafrika ein Potenzial für 165.000 TWh grünen Strom pro Jahr aufzeigt.

Was folgt daraus? Die Staatengemeinschaft sollte Sorge dafür tragen, dass sehr viel von diesem grünen Strom rund um die Erde an den richtigen Stellen produziert wird und zwar zu Kosten, die im internationalen Vergleich in der Letztnutzung nicht viel höher liegen als die heutigen Kosten in der Energienutzung. Letztlich müssen dieser grüne Strom und seine Folgeprodukte (wie grüner Wasserstoff) die heutigen Primärenergieträger Kohle, Gas und Öl weitgehend ablösen.

Vernünftigerweise sollte der grüne Weg primär über Kostenargumente, nur sekundär über temporäre öffentliche Förderung, so attraktiv gestaltet werden, dass $u$. a. die entsprechenden, aus dem Bereich der fossilen Energien kommenden Konzerne, Industrien und Staaten ihr Geld nicht mehr in die Exploration von fossilen Energieträgern stecken (bislang etwa $600 \mathrm{Mrd}$. US Dollar pro Jahr), sondern an geeigneten Stellen - vor allem in den Sonnenwüsten der Welt - große Volumina an grünem Strom (und in der Folge auch an grünem Wasserstoff) produzieren - aus dem Grund, dass es sich rechnet.

\section{Grüner Wasserstoff}

Bei vielen Anwendungen wird Energie in einer Form benötigt, die nicht elektrisch ist und zudem losgelöst von einem Leitungssystem eingesetzt wird. Heute spielen in diesem Kontext die fossilen Energieträger eine zentrale Rolle, die teils zur Stromerzeugung, teilweise aber auch in völlig anderer Form (z. B. als Kraftstoffe) genutzt werden. Grüner Wasserstoff eröffnet in diesem Kontext neue Optionen. Deshalb war immer klar und wird immer klarer, dass grüner Wasserstoff als weitere Komponente neben grünem Strom dringend benötigt wird, und zwar in großen Mengen. Das ist 
mittlerweile auch Teil der deutschen Debatte.

Mit dieser Erweiterung der Sicht auf das Thema steht der Weg in eine neue Welt der Energie nicht mehr nur auf dem einen Bein (grüner Strom), sondern auf zwei Beinen (grüner Strom und grüner Wasserstoff), wobei zur Produktion von grünem Wasserstoff sehr große Mengen von grünem Strom benötigt werden, nicht zuletzt wegen der Umwandlungsverluste (etwa $30 \%$ ) im Vergleich zur Direktnutzung von grünem Strom. Grüner Wasserstoff ist also ein Folgeprodukt von grünem Strom, das insbesondere ein großartiger Energiespeicher ist. Viele Anwendungen brauchen eben Energie in einer anderen Form als Strom. Auf diese Weise kommen der grüne Wasserstoff und seine Folgeprodukte ins Spiel.

Aus deutscher Sicht gibt es die Problematik, dass wir uns weder mit grünem Strom noch mit grünem Wasserstoff selber versorgen können - so wie wir uns derzeit auch nicht mit fossilen Energieträgern vor Ort versorgen können. Hinzu kommt, dass grüner Wasserstoff bei uns viel zu teuer ist, um einen weltweit konkurrenzfähigen Einsatz zu ermöglichen. Die Politik adressiert diese Problematik in der deutschen Wasserstoffstrategie mit Förderprogrammen für grünen Wasserstoff (z. B. H2 Global), aber auch mit der Förderung eines grünen „Hochlaufs" in Deutschland.

Aus Sicht des Autors ist die Debatte um grünen Wasserstoff in Deutschland und Europa nach wie vor viel zu sehr bestimmt durch die wenig realistische Idee mancher Akteure, dass die von uns genutzte erneuerbare Energie zu großen Teilen in Deutschland bzw. Europa hergestellt werden soll. Wir haben dafür weder die Flächen noch die richtige Sonneneinstrahlung. Es ist letztlich nicht zu verstehen, warum man so fixiert auf die Produktion der Energieträger vor Ort ist. Denn auch Erdöl, Gas und Kohle werden importiert durchaus zu unserem eigenen Vorteil, denn im Gegenzug wird unsere Technologie exportiert. Unsere Zahlungsbilanz als „Exportweltmeister" beinhaltet seit jeher erhebliche Überschüsse. Das zu ertragen, ist für die Welt schon schwer genug.

\section{Synthetische Kraftstoffe (e-Fuels)}

Grüner Strom und grüner Wasserstoff reichen aber allein für eine weltweite klimaneutrale Zukunft nicht aus. Bei internationaler Herstellung bleiben Transportprobleme, die bei Wasserstoff gravierend sind, insbesondere beim Transport über große Ozeane. Deshalb brauchen wir neben grünem Strom und grünem Wasserstoff noch ein drittes Standbein. Dieses dritte Standbein führt zu den e-Fuels oder auch reFuels (regenerative Kraftstoffe).

Allein wegen der geographischen und klimatischen Verhält- nisse benötigen wir diese dritte Komponente, und zwar als Energieträger, um die Energie speichern und transportieren zu können. Synthetische Kraftstoffe, wie z. B. grünes Methanol, grünes Methan und grünes Ammoniak, sind für vielfältigste Anwendungen geeignet und lassen sich aus grünem Wasserstoff herstellen. Es existieren dafür verschiedene Syntheseverfahren. So gibt es z. B. den direkten Syntheseweg und die FischerTropsch-Linie.

Besonders attraktiv erscheint dem Autor aus verschiedenen Gründen der Technikpfad über die Direktsynthese von Methanol und Methan. Beide Substanzen sind sehr gute Energiespeicher und vergleichsweise einfach transportierbar. Vor allem die Methanolschiene eröffnet ein breites Feld in Richtung vielfältiger Anwendungen. Die grüne Methanschiene führt - wie die Methanolschiene - in Richtung Gasanwendungen, z. B. Wärme/Kälte in Häusern oder (Kohle) Kraftwerken, aber auch zu den Themen Stahl und Zement. Das Thema Wärme/Kälte ist bekanntlich von zentraler Bedeutung und es gibt dafür klügere, vor allem auch preiswertere Lösungen als die ausschließliche Konzentration auf die teure energetische Sanierung von Gebäuden, nämlich der Einsatz von klimaneutralem synthetischem Heizöl.

Von Methanol ausgehend sind die Wege zu synthetischem Benzin, Diesel, Kerosin, Schiffsbenzin sowie Heizöl vielversprechend. Es sind diese reFuels, die helfen, unsere Zivilisation zu tragbaren Kosten in Richtung Klimaneutralität umzubauen. Die Rezyklierung von $\mathrm{CO}_{2}$ aus Industrieanlagen und Kraftwerken und die $\mathrm{CO}_{2}$ Nutzung zur Produktion der synthetischen Kraftstoffe spielt dabei eine große Rolle. Das ist ein großer Vorzug des hier beschriebenen Weges.

Man hat über solche reFuels insbesondere eine realistische Option, den Gesamtbestand an Fahrzeugen mit Verbrennungsmotoren weltweit in Richtung Klimaneutralität zu bewegen. Dabei spielt die Individualmobilität eine zentrale Rolle, ebenso individuelles Heizen und Kühlen. Im Mobilitätsbereich geht es, wie oben schon erwähnt, weltweit um etwa 1,3 Mrd. Fahrzeuge, die insgesamt pro Jahr etwa 5 Mrd. Tonnen $\mathrm{CO}_{2}$ in die Atmosphäre entlassen. Das ist fast das Doppelte der $\mathrm{CO}_{2}$-Emissionen innerhalb der EU.

Insgesamt bettet sich der beschriebene Weg in ein seit Jahrzehnten bearbeitetes Umfeld ein, für das auch der Begriff einer Methanolökonomie verwendet wird. Methanol ist heute (allerdings in "schwarzer" Form), die am zweit häufigsten synthetisierte Energieflüssigkeit weltweit. Weltmarktführer ist China. Methanol aus Kohle dient dort dazu, die Importerfordernisse bei Öl zu senken.

\section{Der Verein Global Energy Solutions e. V.}


Der Verein Global Energy Solutions e.V. nimmt sich des Themas in sehr grundsätzlicher Weise an. Der verfolgte Ansatz setzt einerseits auf sehr große Volumina an klimaneutralem Strom, vor allem aus den Sonnenwüsten der Welt. Ein Großteil dieser elektrischen Energie wird zur Herstellung von klimaverträglichem Wasserstoff genutzt. Andererseits setzt der Ansatz auf die massive Nutzung eines klimaverträglichen Einsatzes von Erdgas - letzteres vor allem wegen einer erwarteten Enge an Elektrolyseuren für die Wasserstoffherstellung bis zum Jahr 2050 und darüber hinaus. Anfallendes $\mathrm{CO}_{2}$ wird entweder über CCU oder CCS neutralisiert. Oder, wo CCU oder CCS nicht möglich sind, über additive Nature-based Solutions der Atmosphäre (zur Erreichung einer bilanziellen Klimaneutralität) wieder entzogen. Mit diesem Ansatz, vor allem unter Nutzung synthetischer Kraftstoffe, kann der Verkehrssektor klimaneutral gestellt werden. Das gilt insbesondere für die große weltweite Bestandsflotte mit mehr als 1,3 Milliarden Fahrzeugen. Viele ergänzende Informationen finden sich im Webauftritt www.global-energy-solutions.org, insbesondere auch ein One-Pager, der den Ansatz gesamthaft darstellt. 